\title{
Dissimilarities as indices of individual perceptual structure
}

PAUL D. ISAAC ${ }^{2}$

THE OHIO STATE UNIVERSITY

\begin{abstract}
The generality of dissimilarity judgments and of configurations obtained by multidimensional scaling (MDS) as indices of perceptual structure was examined. The Ss made magnitude estimates of dissimilarity of pairs of photos, and MDS configurations were constructed separately for each $S$. The compatability of dissimilarities (judgments or distances in MDS configurations) with two other tasks Ss performed was then examined. These additional tasks, an "encoding" and a "decoding" task, required the $S$ to first construct a message by making use of a set of four photos and, a week later, to decode the messages. Results obtained were interpreted as indicating a degree of generality of the dissimilarities and supporting a hypothesis of individual differences in perceptual structure for the stimulus set. A "data-purification" hypothesis for MDS was discussed and examined.
\end{abstract}

This study examines dissimilarity judgments and multidimensional configurations as indices of perceptual structure. The general proposal is that if these judgments and configurations are indices of perceptual structure, they should be compatible with performance on other related tasks that call upon this structure. This hypothesis of compatibility is examined by making within-S predictions of responses on tasks differing somewhat from the initial dissimilarity judgments. In addition, the procedures used make it possible to examine the hypothesis that differences between $S s$ in dissimilarity judgments reflect perceptual differences. Finally, a "data-purification" hypothesis for multidimensional scaling (MDS) is indirectly tested.

Similarity data have been proposed as a means by which perceptual and cognitive structure can be examined (Coombs, 1964, p. 431). In this context, similarity or "psychological distance" is sometimes used to reconstruct a "psychological space" by means of various Euclidean computational techniques (Coombs, 1964; Kruskal, 1964a, b; Lingoes, 1965, 1966; Shepard, 1962; Torgerson, 1958). However, with few exceptions (Cliff \& Young, 1968; Rosenberg, Nelson, \& Vivekananthan, 1967), convergent validation of similarity data or MDS configurations as perceptual indices has been lacking. Thus, this study examines the compatibility of dissimilarity judgments and MDS configurations with performance on related tasks that differ in contextual and response constraints from the original dissimilarity judgments.

This study also examines the possibility that differences between Ss in dissimilarity judgments reflect perceptual differences. Results of preliminary experimentation indicated that the correlations between two sets of similarity judgments obtained from the same $S$ a week apart are greater than the correlations between two sets of judgments from different Ss. The question arises as to whether this kind of stability reflects within-S perceptual consistencies (and between-S perceptual differences), or simply reflects consistencies specific to the dissimilarity judgments. Previous MDS studies have found individual differences in configurations (Helm \& Tucker, 1962; Indow \& Uchizono, 1960; Silver, Landis, \& Messick, 1966). However, the question of whether or not these differences are perceptual was not independently tested (except for Helm and Tucker's color-deficient Ss). The procedures used in this study provide such a test.

The final hypothesis that is of some interest is whether or not MDS is a kind of data-purification procedure. That is, do the relations between points in a multidimensional configuration represent the perceived structure of the stimulus set better than do the original, somewhat noisy, dissimilarity judgments. Kruskal (reported in Shepard, 1966) carried out a Monte Carlo examination of this hypothesis, using artificial data with random noise added. His MDS program (Kruskal, 1964) did recover the true configuration even at "a moderately high level of added "noise." "The present study examines this "data-purification" hypothesis in an indirect way with real data.

\section{Subjects}

Six male and eight female undergraduate students, drawn from the $S$ pool of an introductory psychology course, participated in the experiment. Each $S$ served in two separate sessions 1 week apart.

\section{Stimuli}

Stimuli were Frois-Wittman Photos 8,
$13,14,29,40,47,50,51,55,57,62$, and 63 , mounted on $3 \times 5$ in. cards (Hulin \& Katz, 1935). These are pictures of facial expressions of one man. The 12 photos used represented a restricted range of expressions-they could probably be described as expressionless or somewhat unhappy. Results of previous studies involving pictures of facial expression as stimuli implied that Ss could be expected to differ in dissimilarity judgments if the range of expressions was restricted (e.g., Engen, Levy, \& Schlosberg, 1958).

\section{Procedure}

Session 1: Dissimilarity judgments. Each $S$ made magnitude estimates of dissimilarity of all pairs of the 12 photos. A starter pair was presented and arbitrarily assigned a value of 100 . Ss were instructed to, in effect, preserve ratios of apparent dissimilarity in their estimates. The magnitude estimates were given verbally, and the $S$ could see only the pair he was judging. The order of presentation of pairs was the same for all Ss. These dissimilarity judgments were used as input to Kruskal's (1964a, b) MDS program.

Session 1: Encoding task. After completion of the dissimilarity judgments, each $S$ performed an encoding task. The motivation for this task was that Ss were to construct messages that would identify a particular photo as being the critical one. But the messages would make use of other photos rather than of verbal descriptions. In this task, the set of 12 photos was laid on the table in front of the S. E picked out a particular photo, called the "target," and the $S$ was to choose 3 other photos from the remaining 11 in such a way that, if these 3 photos were presented together with the target, the target would seem "odd" in the set of 4 . The $S$ was told that an optimal strategy to follow would be to choose 3 photos that were different from the target but similar to one another.

$E$ chose each of the 12 photos, in turn, as target, and S chose "contrast" sets of 3 for each target. Thus, each $S$ constructed 12 "message" sets of 4 photos.

Session 2. One week later, $S$ returned for a second session in which he performed a decoding task. In the decoding task, $S$ was presented with 60 message sets of four photos from which he was to choose the "odd" photo.

In addition to the 12 sets that he had 
constructed himself, $\mathrm{S}$ was given 48 other message sets. Thirty-six of these 48 were sets constructed by three other Ss. (These were called the "other" message sets.) The remaining 12 sets, called "predicted" sets, had been constructed by $E$ on the basis of the S's dissimilarity judgments.

The 12 predicted sets were constructed as follows: Each photo was chosen in turn as target. For each target, a contrast set of three photos was chosen so that (1) it was not the same as the contrast set chosen by $S$ himself, and so that (2) each of the three contrast photos were more similar to each other than they were to the target (according to S's dissimilarity judgments). This is essentially the same strategy that $S$ was told to follow in selecting contrast sets. Occasionally it was not possible to satisfy both criteria, and compromises had to be made. In such a case, the "best"-possible set was chosen.

\section{Basis of Evaluation}

The evaluation of dissimilarity judgments and configurations as perceptual indices is based on a comparison of responses in encoding and decoding with these judgments and with distances derived from the configurations. To avoid unnecessary repetition, "dissimilarities" will be used to refer to both dissimilarity judgments and distances. One question of interest is whether or not contrast sets chosen in the encoding task are compatible with dissimilarities; and the second question here is whether or not choices of "odd" photos in decoding are predictable from dissimilarities.

Recall that the strategy $S$ was told to follow in choosing contrast sets as well as that used in constructing the predicted sets was to choose photos that were different from the target and similar to one another.

The tests of the questions posed above were based on the ranks of the six dissimilarities involved in a message set of four photos. A rank of 1 was assigned to the most dissimilar pair and 6 to the least dissimilar pair. For each photo in a set of four, the sum of ranks of its dissimilarities to the other three in the set can be computed. A S's choice was said to be optimal if the "target" or "odd" photo (in encoding or decoding, respectively) had minimal rank sum of all possible dissimilarity rank sums involving one photo.

Refer to Table 1, which presents hypothetical dissimilarity judgments, with ranks in parentheses. Part (a) illustrates one possible pattern that would be classified as an optimal response. $R$ stands for the target if this message set was encoded and stands for the photo chosen as "odd" if this set had been decoded. Note that the
Table 1

Illustration of Dissimilarity (Rank) HalfMatrices for Message Sets*

\begin{tabular}{|c|c|c|c|c|}
\hline & & A & B & $\mathrm{C}$ \\
\hline (a) & $\begin{array}{l}\mathbf{R} \\
\text { A } \\
\mathbf{B}\end{array}$ & $200^{(1)}$ & $\begin{array}{l}175^{(2)} \\
150^{(3)}\end{array}$ & $\begin{array}{r}125^{(4)} \\
50^{(6)} \\
75^{(5)}\end{array}$ \\
\hline (b) & $\begin{array}{l}\mathbf{R} \\
\mathbf{A} \\
\mathbf{B}\end{array}$ & $125^{(4)}$ & $\begin{array}{l}175^{(2)} \\
200^{(1)}\end{array}$ & $\begin{array}{r}75^{(5)} \\
50^{(6)} \\
\end{array}$ \\
\hline (c) & $\begin{array}{l}\mathbf{D} \\
\mathrm{R} \\
\mathbf{A} \\
\mathbf{B}\end{array}$ & $125^{(4)}$ & $\begin{array}{r}175^{(2)} \\
50^{(6)}\end{array}$ & $\begin{array}{r}150^{(3)} \\
75^{(5)} \\
200^{(1)}\end{array}$ \\
\hline
\end{tabular}

* Message Set: Photos $R, A, B, C$. (Contrast Set: $A, B, C$ )

rank sum of dissimilarities involving the photo, $R$, is minimal of all rank sums involving one stimulus, i.e., rank sum is 7 .

Part (b) illustrates one possible nonoptimal pattern. This case would arise if $A B C$ was the contrast set in encoding or if $\mathrm{R}$ was chosen as odd in decoding. The optimal photo here is $B$, which has a rank sum of 6 . Note that if a $S$ chose $A B C$ in encoding, his encoding would be nonoptimal. But if he chose B in decoding, his decoding would be optimal.

Part (c) illustrates a pattern in which no decision was made. The minimal rank sum here is 9 , but three photos satisfy this minimum, i.e., R, B, C. This kind of pattern was classified as a tie or toss-up.

The actual classification rule is as follows: Encoding (or decoding) is "optimal" if (1) the target (or odd photo) has minimal rank sum of all possible rank sums involving one photo (paired with each of the three others), (2) its rank sum is 9.0 or less, and (3) the next nearest photo has rank sum at least one unit greater. If (1) and (2) are satisfied, but (3) is not, then the set is put into the "toss-up" category. "Nonoptimal" responses constitute the remaining cases.

To the extent that dissimilarity judgments and configurations are indices of the perceptual structure of a set of stimuli for a $S$, the encoding and decoding choices of Ss should be mostly optimal, whether classified by dissimilarity judgments or by distances. Notice that the contexts involved in the encoding and decoding tasks differ somewhat from that of the dissimilarity task, and the responses required of the $S$ are certainly different from the numerical dissimilarity judgments, which can be expected to be rather noisy. (It should be noted that the optimality criterion used here is only one of many possible. But it does seem to capture optimal choices appropriately in terms of a geometric model in which the outlier point is odd.)

If MDS is a kind of data-purification procedure, responses should be classified as optimal more often by distances than by dissimilarity judgments, assuming $S s$ are generally behaving optimally by the above criteria.

If dissimilarity judgments are general indices of perceptual structure for the total set of stimuli, Ss should respond with better than chance accuracy to the predicted sets.

Finally, if Ss do differ from one another in their perceptions of the stimuli, they should decode their own message sets more accurately than those of other Ss. Alternatives to this interpretation are discussed later in the paper.

\section{Design}

The original design called for $12 \mathrm{Ss}$, with Ss being divided into four blocks of three. A S in each block was a decoder of other, own, and predicted messages. Ss who were decoders in one block were to be other encoders in another. Although this design was not quite realized, there were no apparent biases in the resulting design.

\section{RESULTS}

Multidimensional configurations were constructed for all Ss by means of Kruskal's MDS program, using the dissimilarity judgments obtained in Session 1 as input. An attempt was made to avoid the local minimum problem by restarting the program in each of the four dimensions, ranging from 5 to 2 . Note that this procedure gives two four-dimensional configurations, three three-dimensional, and four two-dimensional.) The minimum stress value at each dimensionality was used to plot the stress curve. Optimality classification on the basis of distance (as opposed to dissimilarity) was based on the configuration at which there was a break in the stress curve. The stress value at the break ranged from .048 to .071 , and the dimensionality of the configuration chosen ranged from two to four. (An additional analysis of distances, using more than one configuration for each $S$, is discussed in a later section.)

The Relation of Dissimilarity Judgments and Distances to Encoding and Decoding Tasks

Whether the classification is made on the basis of dissimilarity judgments or on the basis of distances, encoding responses of Ss are mostly optimal, with the proportion of optimal responses being .631 and .597 , respectively (see Table 2). By the optimality criteria described above, if dissimilarities were unrelated to encoding, and there were not ties, the probability of a rank sum of $6,7,8$, or 9 is .19 , giving an expected number of 2.3 optimal sets out of 
Table 2

Optimality of Choices of Contrast Sets in Encoding (Proportions)

(a) Using Dissimilarity Judgments

for Classification

$\begin{array}{ccc}0 & \text { No } & \mathrm{T} \\ 631 & .257 & .111\end{array}$

(b) Using Distances for Classification .597 .292

.111

Probability of optimal ( 0 ) choice if dissimilarities were unrelated to encoding is .19 .

12. The range for individual $S$ s classified either by dissimilarities or distances is 5-11. The results support the hypothesis that dissimiarlity judgments and MDS configurations represent processes that are involved in the encoding task. A data-purification hypothesis is not supported, however, since this hypothesis would require distances to classify responses as optimal more often than do dissimilarity judgments.

Table 3 contains optimality data for decoding. Again it can be seen that the bulk of decoding responses are optimal, whether classified by dissimilarities (a) or by distances (b). The proportion $\mathrm{P}(\mathrm{O} \mid \mathrm{O}$ or NO) refers to the proportion of optimal responses relative to the total number of responses classified as either optimal or nonoptimal (in decoding). The range of scores classified as optimal by either method is: other, 13-29 (36 possible); own, 4-11 (12 possible); and predicted, 5-12 (12 possible).

The results for optimality of encoding and decoding support the proposal that similarity (dissimilarity) judgments and configurations are somewhat more general indices of perceptual structure, even though the judgments themselves may be rather noisy. That results based on distances are similar to those based on dissimilarity judgments might have been expected from the fact that configurations with relatively low stress values were used. However, it is conceivable that local characteristics of the space might differ

Table 3

Optimality of Choices of "Odd" Stimuli in Decoding (Proportions)

\begin{tabular}{|c|c|c|c|c|}
\hline \multirow{2}{*}{$\begin{array}{c}\text { Message } \\
\text { Set }\end{array}$} & \multicolumn{3}{|c|}{ Decoding Optimality } & \multirow{2}{*}{$\begin{array}{c}\mathrm{P}(0 / 0 \\
\text { or } \mathrm{NO})\end{array}$} \\
\hline & 0 & NO & $\mathrm{T}$ & \\
\hline
\end{tabular}

(a) Decoding optimality for "Other," "Own," and "Predicted" message sets, classified by dissimilarity judgments

$\begin{array}{lllll}\text { Other } & .502 & .356 & .141(\mathrm{~N}=432) & .585\end{array}$

$\begin{array}{llll}\text { Own } & .625 & .257 & .118(\mathrm{~N}=144) .709\end{array}$

$\begin{array}{lllll}\text { Predicted } & .680 & .278 & .042(\mathrm{~N}=144) & .710\end{array}$

(b) Same responses classified by distances in configurations

\begin{tabular}{lllll} 
Other & .507 & .380 & .113 & .572 \\
Own & .576 & .292 & .132 & .664 \\
Predicted & .625 & .292 & .083 & .682 \\
\hline
\end{tabular}

from the dissimilarity judgments (for example, in case the scaling of the stimuli in a Euclidean space was inappropriate). This could have led to differences in classification results. It should also be kept in mind that the optimality criterion used here is only one of many which are possible.

Another point of interest is the difference in decoding optimality of other sets relative to that for own and predicted sets. This point will come up again later.

\section{Decoding Accuracy}

As mentioned previously, the procedure used here makes it possible to examine whether or not differences in dissimilarity judgments reflect perceptual differences between Ss. One way to examine the possibility of perceptual differences is to examine the decoding accuracy for the different kinds of message sets. Decoding accuracy for other, own, and predicted sets is presented in Table 4.

Subjects decode their own message sets most accurately (.806) and the predicted sets more accurately than the other sets (.697 vs .581). All differences here are significant $\left[\chi^{2}\right.$ (own, predicted $)=4.4, \chi^{2}$ (own, other) $=23.1$, and $\chi^{2}$ (predicted, other) $=5.81$. Notice that accuracy is defined in terms of identification of the original target as odd in the set.

The dominance of both own and predicted sets over other sets supports the notion of individual perceptual differences. An alternative hypothesis would be that $\mathrm{Ss}$ somehow "remember" their own sets and thus respond to them most accurately. This is unlikely, however, in that Ss decode 60 sets in all, most of which overlap with a number of other sets. However, even if memory could account for own set decoding, it could not account for dominance of predicted sets over other sets, since the predicted sets were selected by $\mathrm{E}$.

An attempt to get an idea of the extent of possible memory effects was made with some Ss. After they had decoded all the message sets in Session 2, they were given some of the sets to identify as being their own or someone else's construction. The results were ambiguous-some $S s$ gave evidence of remembering, others didn't. However, this does not seem to be an appropriate test of a memory hypothesis since Ss had experience with the message sets in decoding prior to the recognition task. Furthermore, even if Ss were able to correctly identify their own sets in this task, it is not necessarily true that they go through this process when they pick out odd stimuli.

The dominance of own sets over predicted sets can probably be attributed
Table 4

Decoding Accuracy

\begin{tabular}{lll}
\hline Message Set & \multicolumn{2}{c}{ Proportion Correct } \\
\hline Other & .581 & $(251 / 432)$ \\
Own & .806 & $(116 / 144)$ \\
Predicted & .697 & $(99 / 142)$ \\
\hline
\end{tabular}

to a number of factors. The optimality criterion is undoubtedly inadequate. Since predicted sets are constructed by using this criterion, some apparently optimal predicted sets may not be optimal, and S would choose the "wrong" odd stimulus. In addition, measurement errors involved in dissimilarity judgments could also result in this kind of misclassification of predicted sets. Finally, compromises sometimes had to be made in construction of predicted sets, since $S$ himself may have chosen the best set in his own encoding. While these factors would affect decoding accuracy of predicted sets, they would not affect decoding of own sets, since $S$ was not constrained by the dissimilarity judgments or previously chosen sets in his encoding.

\section{DISCUSSION}

These results support the hypotheses that similarity judgments and MDS configurations are indices of a more general perceptual structure, and that differences between $S s$ in judgments and configurations can be attributed to individual differences in perception. But there is an anomaly that should be discussed.

Although the bulk of encoding and decoding is optimal, there is a divergence between optimality in decoding other sets and in decoding own or predicted sets. The proportion of optimal choices is somewhat lower in the former case (.502) than in the latter cases (.625 and .680 , respectively). One possible explanation is that there are idiosyncratic encoding and decoding strategies that happen to be incidentally related to dissimilarity judgments; thus, when choices are made from sets constructed by other Ss, the same strategies are not related in the same way to dissimilarity judgments. However, this seems unlikely and ad hoc, and it is not relevant to responses to predicted sets.

Another possibility is that Ss remember the encoded sets, and optimality of decoding simply reflects that of encoding; the proportions of $\mathrm{O}, \mathrm{NO}$, and $\mathrm{T}$ for encoding $(.631, .257$, and .111) are very close to those of decoding $(.625, .257$, and .118 ), so perfect memory is a possible, but unlikely, explanation, as was discussed above with regard to decoding accuracy.

However, neither of these explanations can account for the fact that $\mathrm{P}(\mathrm{O} / \mathrm{O}$ or NO) is equal for predicted and own sets. 
(Comparison of $\mathbf{T}$ choices for predicted and own sets is not really meaningful, since the predicted sets were set up to define an optimal target stimulus, thus minimizing the possibility for $T$ choices-since they could occur only where there wasn't a clearly defined optimal.) Thus, given that S's choice is either $\mathrm{O}$ or NO, according to this statistic, Ss behave in roughly the same manner to own and predicted sets (.709 and .710), both of which differ from the same statistic for other sets (.585). This could not be accounted for by either of the above hypotheses since, as mentioned above, the predicted sets were constructed by $E$ and thus could not be remembered or involve idiosyncratic strategies.

A more likely explanation for these results is that own and predicted sets are decoded more optimally because, on the average, the optimal stimulus is more sharply defined in terms of dissimilarity for these sets than for other sets. This would imply that choices of "odd" stimuli would be relatively more difficult than for own sets and subject to random variation among alternatives which are nearly equally likely.

This explanation could account for the differences between own and other sets with respect to optimality of decoding and also would be consistent with differences in decoding accuracy for these sets. To elaborate, suppose that all of a S's decoding (and encoding) responses were truly optimal. However, the classification scheme used is subject to measurement error and, thus, to misclassification. For message sets that do not have a sharply defined optimal stimulus, measurement errors are more likely to lead to misclassification of optimality than in cases with a sharply defined optimal stimulus. Thus, even if $S$ is behaving optimally, choices of odd stimuli for other sets should be misclassified as nonoptimal more often than for own sets, since the other sets hre hypothesized to have a less sharply defined optimal stimulus.

Results on decoding accuracy lend credence to this argument. A more sharply defined optimal stimulus would lead to greater accuracy in decoding own as opposed to other sets. This, indeed, is the case.

The strongest support for the legitimacy of similarity judgments as perceptual indices and for an individual-differences interpretation comes from the data on predicted sets. These sets were constructed by $E$ on the basis of $S$ 's dissimilarity judgments and were chosen to be different from those $S$ himself constructed (an overlap of two of the three contrast photos was permitted). Since $S$ had not seen these sets before, his choices could not be dictated by perfect memory of selection of the contrast set. The fact that these sets are decoded more accurately than would be expected by chance $(p=.25$, by chance) supports the hypothesis that similarity judgments are legitimate perceptual indices; the fact that they are decoded more accurately than other sets supports a hypothesis of individual differences in perception.

\section{An Additional Test of the "Data-Purification" Hypothesis}

None of the results presented above were consistent with a data-purification hypothesis. That is, responses classified by distances were not optimal more often than responses classified by dissimilarities. This was an admittedly weak test of this hypothesis: The optimality criterion may be wrong or irrelevant, and the configurations used may be inappropriate, either because of dimensionality or because of local minima.

However, assuming that the optimality criterion is acceptable, recall that the configuration used was one where there was a break in the stress curve. A more sensitive test of data purification might be based on the following considerations:

Configurations that fit the data very well may not be the best representation of the perceived structure of the stimulus set since they recapture the noise in the judments as well as the "true" perceived dissimilarity. On the other hand, configurations with "large" stress values might not be representing anything very well. Thus, the true configuration should lie somewhere between those having large and those having small stress values.

Applying this argument to the present situation, it would be hypothesized that optimality classification (i.e., proportion of optimal responses) should be an inverted U-shaped function of stress (or dimensionality). At its peak, at least, this inverted U-shaped function should exceed the proportion of optimal responses $[p(O)]$ as classified by dissimilarity judgments.

This hypothesis was tested in the present decoding data by looking at patterns for individual Ss. No clear-cut results were apparent. Although a number of classifications based on distances did exceed $p(O)$ based on dissimilarity judgments ( 34 of 80 possible), for only one $S$ (of 12) did $\mathrm{p}(\mathrm{O})$ reach a maximum value for both own and other sets using the same configuration (and thus stress and dimensionality) so that the maximum exceeded $p(O)$ from dissimilarity judgments. For four Ss, there was at least one configuration that did as well or better than dissimilarity judgments for both own and other sets, even though there may have been another configuration that did better for own or other sets by themselves.

These data do not clearly support a data-purification hypothesis. However, this was not a very powerful test of the hypothesis. Kruskal's results, mentioned earlier, using artificial data, indicate that for data generated by a known configuration, the MDS procedure will recover it. However, except for the stress criterion and a criterion of "interpretability of dimensions," there is no good way to decide on the dimensionality of a configuration resulting from real data. The approach suggested here is that an independent criterion may be useful, based on other responses of the $S$ himself rather than simply on the interpretations of the $\mathrm{E}$. However, the results do not warrant enthusiasm over the present procedure. The assumption that $S$ is always behaving optimally is suspect, in addition to the adequacy or relevance of the optimality criterion and the possibility that local minima may cause problems as mentioned above. An alternate procedure involving independent rating of dimensions has been used by Rosenberg et al (1967). Also, Cliff and Young (1968) related multidimensional configurations to unidimensional judgments in a slightly different way.

Another approach to studying the data-purification hypothesis and, implicitly, the problem of appropriate dimensionality involves replicated judgments. Such a study is being carried out at present and results will be reported in another paper. In addition, the hypothesis of an inverted U-shaped function may be worth studying with artificial data in this context.

\section{CONCLUSION}

This study supports the hypothesis that dissimilarity judgments and MDS configurations reflect a perceptual structure for the stimulus set that is more general than one simply generating dissimilarity judgments. This was indicated by the compatibility of judgments and configurations with responses in encoding and decoding tasks that differed from the original dissimilarity judgments. Decoding of message sets constructed on the basis of a S's dissimilarity judgments (the predicted sets) gave the strongest support for dissimilarity judgments as perceptual indices and for individual differences in perception.

A data-purification hypothesis was not supported. Optimality classification by distances in configurations at a break in the stress curve was not "better" than that based on the original dissimilarity judgments. A more powerful test of this 
hypothesis was described, but again the results were not convincing. The inadequacy of this test of "data purification" was pointed out.

\section{REFERENCES}

CLIFF, N., \& YOUNG, F. W. On the relation between unidimensional judgments and multidimensional scaling. Organizational Behavior \& Human Performance, 1968, 3, 269-285.

COOMBS, C. H. $A$ theory of data. New York: Wiley, 1964.

ENGEN, T., LEVY, N., \& SCHLOSBERG, H. The dimensional analysis of a new series of facial expressions. Journal of Experimental Psychology, 1958, 55, 454-458.

HELM, C. E., \& TUCKER, L. R. Individual differences in the structure of color-perception. American Joumal of Psychology, 1962, 75, 437-444.

HULIN, W. S., \& KATZ, D. The Frois-Wittman pictures of facial expressions. Journal of Experimental Psychology, 1935, 18, 482-498.
INDOW, T., \& UCHIZONO, T. Multidimensional mapping of Munsell colors varying in hue and chroma. Journal of Experimental Psychology, $1960,59,321-329$.

KRUSKAL, J. B. Multidimensional scaling by optimizing goodness of fit to a nonmetric hypothesis. Psychometrika, 1964a, 29, 1-27.

KRUSKAL, J. B. Nonmetric multidimensional scaling: A numerical method. Psychometrika, 1964 b, 29, 115-130

LINGOES, J. C. An IBM-7090 program for Guttman-Lingoes smallest space analysis $-I$. Behavioral Science, 1965, 10, 183-184.

LINGOES, J. C. An IBM-7090 program for Guttman-Lingoes smallest space analysis-RII. Behavioral Science, 1966, 11, 322.

ROSENBERG, S., NELSON, C., \& VIVEKANANTHAN, P. S. A multidimensional approach to the structure of personality impressions. Mimeo, Rutgers University, 1967. SHEPARD, R. N. The analysis of proximities: Multidimensional scaling with an unknown distance function. I. Psychometrika, 1962, 27 , 127-140.

SHEPARD, R. N. Metric structures in ordinal data. Journal of Mathematical Psychology, $1966,3,287-315$

SILVER, C. A., LANDIS, D., \& MESSICK, S. Multidimensional analysis of visual form: An analysis of individual differences. American Journal of Psychology, 1966, 79, 62-72.

TORGERSON, W. S. Theory and methods of scaling. New York: Wiley, 1958.

\section{NOTES}

1. The research reported here was supported by United States Public Health Service Grant GM 01231. The paper is based on the author's doctoral dissertation, completed at the University of Michigan in 1968. The author expresses his appreciation to the members of the Doctoral Committee, chaired by David $\mathrm{H}$ Krantz, for their helpful suggestions and comments.

2. Address: Department of Psychology, The Ohio State University, Columbus, Ohio 43210.

(Accepted for publication May 28, 1969.) 